\title{
Dangling bond detective work
}

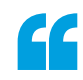

\section{Our calcula- tions of the properties of dangling} bonds showed that they were consistent with previous measurements of single-photon emission.

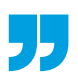

Single-photon sources are

key for a number of quantum technologies. They enable quantum communication, and are essential for the proposed implementations of light-based quantum computers: either photons are used to entangle two quantum bits (qubits), or photons themselves act as 'flying qubits'.

Although single-photon emission has been detected in various $2 \mathrm{D}$ materials, including hexagonal boron nitride ( $\mathrm{h}-\mathrm{BN})$, its mechanism is not well understood. Writing in Physical Review Letters, Chris Van de Walle and colleagues present density functional theory calculations which correlate single-photon emission with particular defects in the h-BN lattice.

"By thoroughly analysing the reported results on single-photon emitters in h-BN, we aimed to identify a defect or impurity that

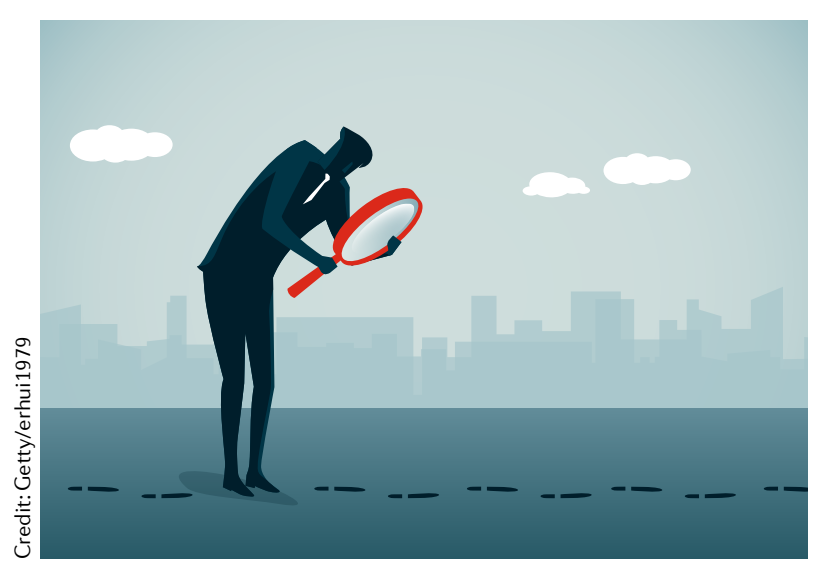

could explain all of the observed properties. This process led us to consider dangling bonds," says Van de Walle. Dangling bonds are formed when the crystal structure is disrupted, for example, at a surface or a grain boundary.

"Dangling bonds are an often overlooked defect, despite being present in most materials" explains Van de Walle. "Our calculations of the properties of dangling bonds showed that they were consistent with previous measurements of single-photon emission." These observations show that single photons emitted from $\mathrm{h}-\mathrm{BN}$ are linearly polarized, and have energies of approximately $2 \mathrm{eV}$ with a sharp zero-phonon line.

The researchers model an h-BN lattice comprising defects and characterize the properties of isolated nitrogen or boron dangling bonds. The calculations show that nitrogen dangling bonds do not give rise to strong optical emission whereas the boron dangling bonds can emit single photons. Given that most h-BN flakes are nitrogen-terminated, boron dangling bonds are relatively rare, which matches the infrequency of single-photon emission in experimental data.

Modelling of the boron dangling bond reveals two energy levels - only one of which is occupied - within the bandgap of the material. A transition between these energy levels emits a single photon, but its energy is over $3 \mathrm{eV}$, which does not match the data. In practice, oxygen impurities are likely to occur in h-BN; they act as donors and provide electrons that cause the boron dangling bond to become negatively charged. The model of the charged dangling bond shows a different transition, which does have an energy of $2 \mathrm{eV}$ and a sharp zero-phonon line.

By tweaking the symmetries of the system further, the team found that the wavelength of the emitted photons is sensitive to small distortions in the lattice. In practice, the defects which have dangling bonds are likely to occur near flake edges and grain boundaries. This local lattice distortion would explain why a wide range of wavelengths of single photons have been observed.

Understanding the microscopic origin of this mechanism opens up the possibility to control it in a more useful way. "We hope these results draw attention to the important role of dangling bonds, so that more directed experimental techniques can be applied to control the presence and properties of dangling bonds," concludes Van de Walle. "We also plan to explore the potential of dangling bonds in other materials for single-photon emission."

Ankita Anirban

ORIGINAL ARTICLE Turiansky et al. Dangling bonds in hexagonal boron nitride as single-photon emitters. Phys. Rev. Lett. 123, 127401 (2019) 